\title{
BARRERAS PERCIBIDAS POR LOS USUARIOS PARA ACCEDER AL TEST DE ELISA PARA VIH ${ }^{1}$
}

\section{UNCOVERING THE PERCEIVED BARRIERS OF USERS TO ACCESS TO TEST FOR HIV ELISA TEST}

\author{
Paula Vega Vega* \\ Alejandra-Ximena Araya G. ${ }^{* *}$ \\ María-Teresa Urrutia Soto ${ }^{* * *}$ \\ Paola Carrasco Aldunate ${ }^{* * *}$ \\ Miriam Rubio Acuña ${ }^{* * * *}$
}

\begin{abstract}
RESUMEN
Objetivo: Develar las barreras percibidas por los usuarios con serología desconocida para acceder al test de Elisa, para el diagnóstico de VIH a nivel de Atención Primaria de Salud. Material y método: Estudio cualitativo descriptivo exploratorio en el que se realizaron 30 entrevistas en profundidad a usuarios con serología desconocida para Virus de la Inmunodeficiencia Humana (VIH) de dos centros de salud, quienes solicitaron el examen por iniciativa propia. Las entrevistas fueron grabadas y transcritas textualmente, con previo consentimiento de los participantes. El análisis de contenido se realizó según Kripperdorff y se cumplieron los criterios de rigor según Guba y Lincoln. Resultados: Del análisis emergieron 3 dimensiones que corresponden a las barreras: Temor por la connotación negativa del examen del VIH, escasa información entregada por el personal de los centros y burocracia en el proceso de toma del examen del VIH. Conclusión: Las barreras percibidas por los usuarios aparecen desde sus propios temores y de aspectos administrativos de los centros de salud, dificultando el acceso voluntario a la toma del examen del VIH.
\end{abstract}

Palabras clave: Test de Elisa, accesibilidad a los servicios de salud, VIH.

\begin{abstract}
Objective: Reveal the perceived barriers by users with unknown serology to access the Elisa test for HIV diagnosis. Method: A qualitative and descriptive study was undertaken through 30 in depth interviews to patients with unknown HIV status of two primary health care centers, which ones had request the test by themselves. The interviews were audiotaped and transcribed verbatim after gaining the consent of the participants. The content analysis was performed according to Kripperdorff and was ensured the rigor criterion according Guba and

\footnotetext{
${ }^{1}$ Proyecto financiado por el Fondo Nacional de Investigación en Salud, CONICYT-MINSAL, CHILE. Proyecto FONIS No SA11I2137.

* Enfermera Matrona, Master of Science, Profesor Asociado. Escuela de Enfermería, Pontificia Universidad Católica de Chile. Vicuña Mackenna 4860. Macul. Santiago; E-mail: pvegav@uc.cl

${ }^{* *}$ Enfermera Matrona, PhD, Profesor Asociado, Escuela de Enfermería, Pontificia Universidad Católica de Chile. E-mail: aarayau@uc.cl

${ }^{* * *}$ Enfermera Matrona, PhD, Profesor Asociado, Escuela de Enfermería, Pontificia Universidad Católica de Chile. E-mail: murrutis@uc.cl

${ }^{* * * *}$ Enfermera Matrona, Profesor Asistente, Escuela de Enfermería, Pontificia Universidad Católica de Chile. E-mail: dcarrasa@uc.cl

${ }_{* * * *}^{*}$ Enfermera, Profesor Asistente, Escuela de Enfermería, Pontificia Universidad Católica de Chile. E-mail: merubioa@uc.cl
} 
Lincoln. Results: Three dimensions were identified through content analysis that correspond to the barriers: Fear for negative connotation of HIV test, Limited information provided by the center staff and Bureaucracy in the process of taking the HIV test. Conclusion: The barriers perceived for the patients appears by their own fears and by administrative aspects of the health centers, making difficult the voluntary access to undertake the HIV screening test.

Key words: ELISA, health services accessibility, HIV.

Fecha recepción: 27/08/14 Fecha aceptación: 06/06/15

\section{INTRODUCCIÓN}

En Chile, la aplicación de políticas públicas de salud, ha mejorado considerablemente el acceso y disponibilidad a la toma del examen del test de Elisa para el diagnóstico del Virus de la Inmunodeficiencia Humana (VIH), gracias a su gratuidad y a su disposición en los Centros de Salud Familiar (CESFAM), a nivel de la Atención Primaria de Salud (APS) nacional $(1,2)$.

A pesar de esto, existe un importante número de personas que se toman tardíamente el examen y, por lo tanto, acceden en etapas avanzadas de la enfermedad al tratamiento con terapia antirretroviral (3). El diagnóstico tardío del VIH es un problema mundial, con cifras que van entre un 22 y un $83 \%$ de personas que viven con VIH (PVVIH) y fueron diagnosticadas tardíamente tanto en Europa como en los Estados Unidos $(4,5)$. En el caso de Chile, esto corresponde al $46 \%$ de las PVVIH (6).

Varios estudios han demostrado que el diagnóstico oportuno tiene beneficios tanto a nivel individual como colectivo (7), aumentando la tasa de sobrevida y disminuyendo la tasa de morbilidad asociada a la enfermedad (8-10).

Marks et al. (11) señalan que la epidemia del VIH/SIDA puede ser substancialmente disminuida si se aumenta el número de personas que conocen su serología positiva, dado que la tasa de transmisión es 3,5 veces mayor en el grupo de personas que no conocen su diagnóstico de VIH. Por esto se hace necesario expandir el acceso a la toma del test de Elisa, y así aumentar el número de personas que conocen su estado.

Existen pocos estudios que han explorado las barreras, motivaciones y/o razones para la toma oportuna del examen del VIH, encontrándose que el retraso en la toma del test se debe al miedo al diagnóstico, a la carencia de seguro de salud, a la falta de conocimiento sobre la terapia antirretroviral, al alto uso de sustancia ilícitas y a la falta de percepción de riesgo de adquirir la enfermedad (7). Sin embargo, en Chile no existen estudios publicados sobre las dificultades a las cuales se ven expuestos los usuarios de APS que desean acceder a este examen.

El objetivo del presente estudio es develar las barreras percibidas por los usuarios con serología desconocida para acceder al test de Elisa, para el diagnóstico de VIH a nivel de APS.

\section{MATERIAL Y MÉTODO}

Para el presente estudio se utilizó la metodología Cualitativa Exploratoria (12) en base al análisis de contenido de entrevistas planteado por Krippendorff (13). Este estudio se realizó a 30 usuarios con serología desconocida para VIH de 2 CESFAM del Servicio de Salud Metropolitano Sur Oriente, que solicitaron 
el examen del test de Elisa en forma espontánea. Los participantes fueron invitados por los profesionales a cargo de la consejería de cada centro de salud, entre los meses de marzo y junio del año 2012. Los criterios de inclusión fueron: personas mayores de 18 años, que espontáneamente solicitaron el examen VIH en el CESFAM y que voluntariamente aceptó participar. Se excluyeron embarazadas y personas en proceso de donación de sangre, dado que en ambos casos no accedieron al examen por consulta espontánea. Los participantes interesados fueron contactados telefónicamente por una de las investigadoras, para acordar la fecha y lugar de la entrevista. Se realizó una entrevista en profundidad, cuya pregunta central fue: ¿Cuál o cuáles han sido las dificultades que usted ha tenido para tomarse este test en este CESFAM? Las entrevistas fueron audiograbadas previa firma del consentimiento informado. Una vez finalizada la entrevista, se aplicó un cuestionario para recolectar variables sociodemográficas y de salud. Las entrevistas duraron en promedio 40 minutos y fueron transcritas textualmente, manteniendo la confidencialidad de los datos.

El análisis de los datos se realizó a través del análisis de contenido cualitativo inductivo (13). Para ello, cinco investigadoras previamente capacitadas y en base a las entrevistas transcritas realizaron, cada una por separado, el análisis de las entrevistas: primera lectura fluctuante de las narrativas, segunda lectura para identificar las dimensiones y categorías, tanto explícitas como implícitas, y una tercera, para ratificar las categorías o dimensiones encontradas. Posteriormente, las investigadoras se reunieron para llegar a un consenso con respecto a las categorías y dimensiones develadas. Se aplicó el criterio clásico de la saturación de significados, el cual se logró con 12 entrevistas por CESFAM, sin embargo el equipo investigador realizó 3 entrevistas más para confirmar los hallazgos.

Para asegurar la validez de los resultados se utilizaron los criterios de rigor metodo- lógico de Guba y Lincoln (14). La credibilidad se logró tras realizar la devolución de las categorías y dimensiones a 10 de los participantes por centro, quienes se sintieron reflejados con los hallazgos. La confiabilidad se logró tras la realización de las reuniones de consenso y el uso de narrativas. La confirmabilidad, a través de una descripción detallada de la investigación en un informe y la transferibilidad se obtuvo a través de la validación de los hallazgos por 3 expertos. Los datos cuantitativos se analizaron a través de medidas de tendencia centrales.

Para resguardar el cumplimiento de los aspectos éticos de la presente investigación se utilizaron los siete aspectos de Emmanuel et al. (15). Además de contar con la aprobación del Comité de Ética de la Escuela de Enfermería de la Pontificia Universidad Católica de Chile y del Comité de Ética del Área Sur Oriente de la Región Metropolitana.

Una de las limitaciones de este estudio es que sólo se pudo acceder a aquellos usuarios que espontáneamente solicitaron el examen, y por lo tanto tuvieron acceso al proceso de toma del test, sin embargo existen personas que no concurren a los centros o no han logrado solicitar el examen, y por consiguiente no se pudo comprender su vivencia.

\section{RESULTADOS}

\section{Característica de los participantes:}

De los 30 usuarios con serología desconocida, 23 fueron mujeres y 7 hombres. El rango de edad fluctuó entre los 19 y 77 años, con una media de 34 años. En relación a su orientación sexual, 2 de los participantes declararon ser homosexuales; uno, bisexual, y 27, heterosexuales. De los 30 participantes, 4 estaban casados al momento de la entrevista, 21 solteros, 4 separados y una persona era viuda. De todos ellos, 22 declararon tener 
hijos. Con respecto al nivel educacional, 10 de los participantes tenía enseñanza básica completa, 10 enseñanza media completa y 4 , estudios técnicos. Del total de participantes, 17 tenían trabajo estable (jornada completa o media jornada), 5 trabajaban en forma independiente, 5 estaban sin empleo al momento de la entrevista y el resto tenían trabajos esporádicos. En cuanto a la previsión de salud, los 30 participantes pertenecían al sistema estatal correspondiente al Fondo Nacional de Salud (FONASA). Respecto a factores de riesgo, los 30 participantes declararon no ser consumidores de drogas, sin embargo 10 eran consumidores de alcohol. Del total de participantes, 27 habían tenido relaciones sexuales los últimos 3 meses y de ellos, 12 habían usado preservativos al momento del acto sexual, considerando que 8 de los parti- cipantes refirieron haber estado con más de una pareja.

\section{Hallazgos}

Tras el análisis de las narrativas, emergieron 3 dimensiones con sus categorías, que dieron respuesta al fenómeno: Temor por la connotación negativa del examen del VIH (temor a ser estigmatizados socialmente, miedo a ser discriminados y miedo a que el resultado sea positivo), escasa información entregada por el personal de los CESFAM (escaso acceso a información para la toma del examen y poca información sobre la enfermedad) y burocracia de los CESFAM en el proceso de toma del examen del VIH (demora en la asignación de horas de atención y tardanza en la obtención del resultado) (Figura 1).

Figura 1. Barreras percibidos por los usuarios respecto al acceso al test de Elisa para VIH a nivel de Atención Primaria de Salud.

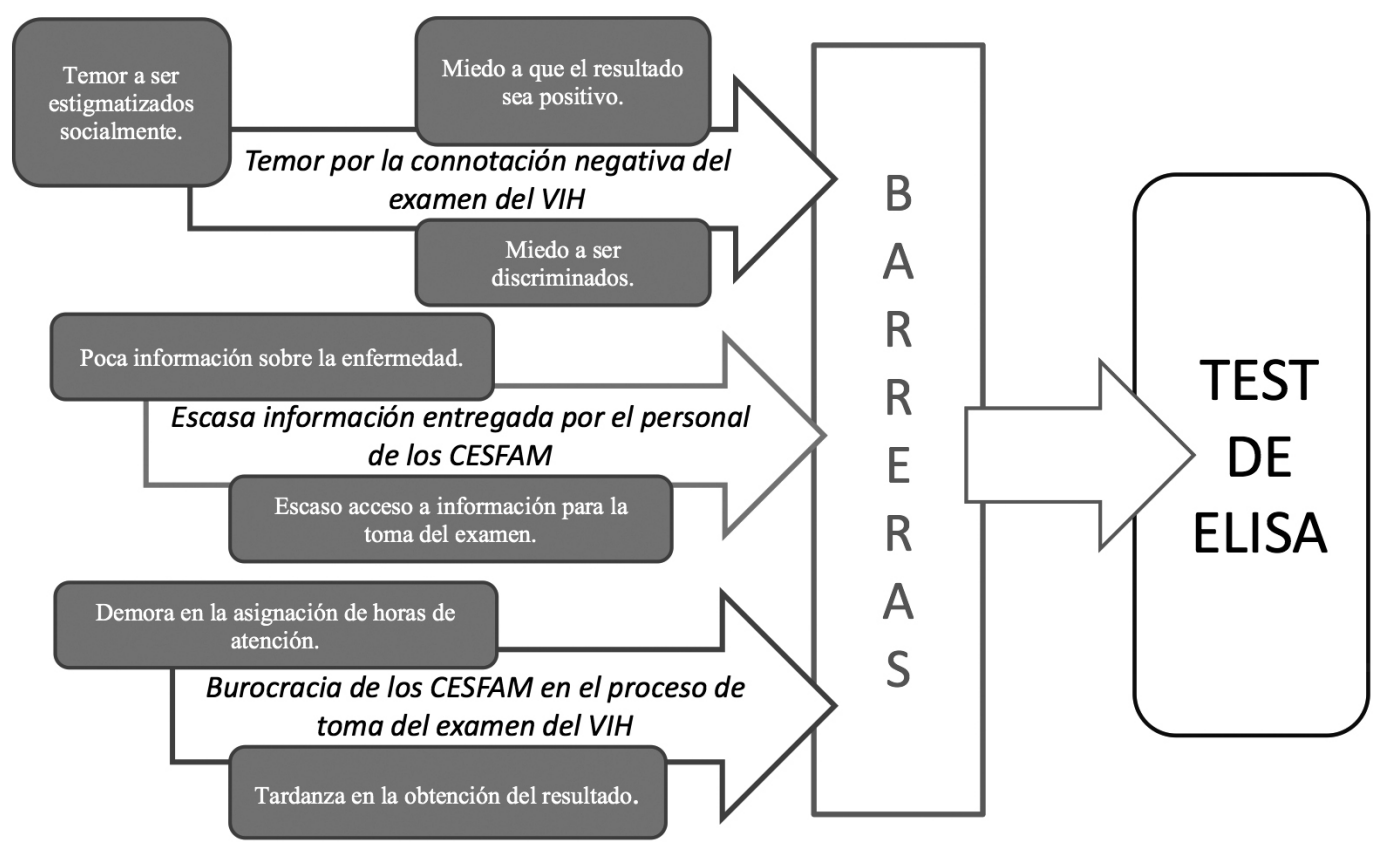




\section{TEMOR POR LA CONNOTACIÓN NEGATIVA DEL VIH}

Para los participantes la connotación negativa que tiene el VIH y su examen diagnóstico, les genera un gran temor de ser estigmatizados socialmente, no solo por el resultado, sino también por el hecho de solicitar el examen. Esto probablemente se explica debido a la carga social que tiene esta enfermedad, con el consiguiente miedo a ser discriminado por los funcionarios que toman el examen o por otros usuarios del mismo CESFAM, que comparten la atención en el mesón al momento de solicitar el examen.

Por otra parte, los usuarios temen a que el resultado del examen del VIH sea positivo, por lo que prefieren no hacerse el examen del VIH y vivir con la duda de su diagnóstico, y de esa manera evitar enfrentar el estigma por estar contagiados.

\section{Temor a ser estigmatizados socialmente:}

Si es que donde uno tiene que ir [mesón de atención], para poder decir vengo a tomarme el examen... en ese momento hay demasiada gente, ¿me entiende?... alrededor es gente de las mismas poblaciones donde uno vive. Entonces si hay un vecino o una persona que te conoce, $y$ tú vas y dices: "Vengo a tomarme el examen del VIH"... y te escuche esa persona, esa persona lo va a divulgar en todas partes.

\section{Miedo a ser discriminado}

Por precaución... Es como llegar y decirlo a viva voz, porque capaz que se mal interprete... "Ah! él sí tiene SIDA"... Entonces yo ahí mejor trato de no, que nadie sepa. Porque pueden pensar, antes de que me hayan hecho el examen... que yo lo tengo, y ya me van a tratar... me van a discriminar.

\section{Miedo a que el resultado sea positivo}

... A mí me da miedo, yo lo reconozco. Como que a mí me da miedo que me dijeran: "Sí, usted tiene esta enfermedad". ;Claro!, las complicaciones de hecho, pero yo creo que todos tenemos el miedo a que nos digan: "¡Tú lo tienes!”.

\section{ESCASA INFORMACIÓN ENTREGADA POR EL PERSONAL DE LOS CESFAM}

Para los usuarios, una de las mayores barreras es la escasez de información con respecto al acceso para la toma del examen que existe a nivel de los CESFAM, desde el cómo, dónde, a quién solicitarlo, y hasta quienes deben tomárselo. Esto repercute en una tardanza en la solicitud de este tipo de examen. A su vez, sostienen que el problema radica en la existencia de poca información en relación a la enfermedad, considerando aspectos como prevención, mecanismo de transmisión y síntomas, lo que repercute en baja percepción de riesgo por parte de la comunidad.

\section{Escasa información sobre el acceso para la toma del examen}

...Tampoco es un examen que se ofrezca. Eso también es un tema importante creo yo... que tal vez hay poca información. Es un tema tabú, que la gente le tiene miedo, que evade... Entonces, pienso que una traba podría ser eso, la falta de información. Pienso que siempre va a ser la falta de información o de educación. O sea, hay un tema como de no darle importancia a ciertas cosas y... yo creo que eso influye muchísimo en que la gente no lo haga. Yo creo que hay gente que ni siquiera sabe que el test existe o que se llama test de ELISA, o que... lo que te decía, yo no tenía idea que se daba tanta facilidad. Entonces, es la falta de educación, y de información, de todas maneras, lo que influye...

\section{Poca información en relación a la enfermedad}

En los sectores más pobres la gente es mucho más ignorante. Me parece que mientras no le toque a uno, uno lo ve desde afuera muy lejos. 
No hay ninguna propaganda, no se habla el tema [del SIDA] en la televisión, no se habla el tema en ninguna parte... está como súper tapado y eso es un tema que yo creo que todo el mundo debiera saberlo...

\section{BUROCRACIA DE LOS CESFAM EN EL PROCESO DE TOMA DEL EXAMEN DEL VIH}

Los participantes sostienen que existe una administración ineficiente en relación al proceso de toma de examen, incluyendo la poca claridad de los protocolos y demora en la asignación de horas de atención, tanto para la preconsejería como para la toma del examen, y la postconsejería. Esto lo transforma en un proceso complejo, poco accesible y que produce una tardanza en la obtención del resultado del test de Elisa.

\section{Demora en la asignación de horas de atención}

Él [Médico] me dijo que tenía que venir a la matrona, jo sea!... no era el que me podía derivar al examen. Yo creo que ese punto debería cambiar. Porque si yo llego y tengo, por decirlo así, la fortaleza de pedirle al doctor... porque uno es como enjuiciado en ese momento. Si tengo esa fortaleza, debería él mismo ordenarla, pienso yo. Y ellos deberían estar, como doctores, preparados para decirnos la consejería... Tuve que ir a la instancia de pedir una hora al matrón, que fue como en veinte días más... No fue al tiro tampoco... Yo de hecho lo pensé, yo pensé en no venir y dije: "Pucha, estoy leseando mucho, yo trabajo"... Entonces, tú sales de tú trabajo con una predisposición y llegas acá, y no puedes, $y$ después tienes que volver a venir a ver si es que tienen tiempo para darte una hora $y$ poder hacértelo. Entonces en ese momento es cuando uno flaquea y dice: "Pucha si mejor intento juntar las lucas o me consigo para hacérmelo particular... jahí no tenis que mirarle la cara a nadie!"

\section{Tardanza en la obtención del resultado}

De hecho todavía pedí hora para ver el examen $y$ me dijeron que tenía que venir a pedir hora a fin de este mes porque no hay horas este mes, $y$ para la agenda de agosto se abre a fin de este mes y es como hay que venir otra vez a sacar hora y después otra vez a venir a la hora...

\section{DISCUSIÓN Y CONCLUSIÓN}

Los usuarios con serología desconocida para VIH que participaron en este estudio percibieron una serie de dificultades que les entorpeció acceder en forma expedita a la toma del test de Elisa. Estas barreras, en una primera instancia, están determinadas por el temor que existe frente al VIH y a lo que se relaciona con éste, como es el test de Elisa. Algunos estudios han demostrado que el miedo a ser estigmatizados y discriminados, evita que las personas se atrevan a solicitarlo a los profesionales de la salud (16-19), lo que también fue descrito por los participantes del presente estudio. A sí mismo, el miedo de los participantes a obtener un resultado positivo del test, restringió el acceso al examen, lo que a su vez fue descrito en estudios realizados en USA (20) y Uganda (21). Otra dificultad presentada en los estudios de Spielberg et al. (20) y Mill et al. (22) fue la percepción de invulnerabilidad por parte de los usuarios, sin embargo los participantes del presente estudio no hacen referencia a ello en sus narrativas.

Una segunda instancia que afecta la toma de decisión de solicitar el examen, está dada por falencias que existe en la entrega de contenidos educativos en relación a los factores de riesgo para adquirir el virus del VIH, situación que fue develada en otros estudios $(7,17)$. A su vez, la falta de información y/o desconocimiento de los usuarios sobre la disponibilidad del test en los centros de salud es 
una de las principales barreras que dificultan el acceso al examen $(23,24)$, lo que también fue verbalizado por los participantes del presente estudio.

El tercer elemento a considerar fue la burocracia en el proceso de la toma del examen, lo cual ha sido descrito en otras investigaciones $(7,16)$. Sin embargo, en este estudio la dificultad estaba centrada en la falta de horas/profesional para la consejería previa al examen, según lo dispuesto en las Guías Clínicas del MINSAL (25). Es importante destacar que los usuarios del presente estudio no señalaron como barrera la actitud relacional con los profesionales del centro de salud, situación descrita en estudios realizados en Sud África (17) y Canadá (26), en los cuales los participantes manifestaron abiertamente que la actitud poco empática y acogedora de los profesionales de la salud que los atendieron obstaculizó el acceso al examen. Esto se hace más manifiesto en aquellos casos en los cuales los usuarios se sienten vulnerables frente a la privacidad y confidencialidad de sus datos (27), situación que no fue compartida por los participantes del presente estudio.

Las autoras reconocen que una limitante de esta investigación fue que se estudió a un grupo determinado de usuarios con serología desconocida de un sector preestablecido de la Región Metropolitana, por lo que los hallazgos solo pueden ser trasferidos a poblaciones con similares características. Sin embargo, es el primer estudio en Chile que profundiza en las percepciones de los propios usuarios sobre la problemática en el acceso al test de Elisa para el diagnóstico oportuno del VIH.

Por todo lo anterior, se hace necesario generar nuevas estrategias, a las ya existentes, que optimicen aún más el acceso de la comunidad a la toma del test de Elisa y, por lo mismo, desarrollar instancias de educación formal que permitan a los usuarios tomar decisiones informadas al respecto, y así incentivarlos para que por iniciativa propia soliciten el examen a nivel de APS. A esto se suma el requerimiento de algunos cambios administrativos en los centros de salud, como: el acceso a un mayor número de horas/profesionales que otorguen consejería, a mejorar la privacidad al momento de solicitar el examen en el mesón de atención con las secretarias, implementar la prueba oral rápida para el tamizaje del VIH en APS (28), incorporar el test de Elisa dentro de los exámenes de rutina en el control de salud del adulto con el objeto de normalizar la actitud frente al VIH $(29,30)$, adecuar los horarios para las personas que trabajan, hacer campañas de difusión y educación en la comunidad (juntas de vecinos, escuelas, plazas, ferias libres, etc.) y mantener campañas periódicas dentro de los centros de salud que motiven la toma del examen. Se esperaría que con estas estrategias existiera un mayor número de personas con serología desconocida que por iniciativa propia solicitaran el examen, y con ello, tomar las medidas necesarias para disminuir la cadena de contagio (11).

Es importante señalar que se socializaron los hallazgos del estudio con ambos CESFAM, con el objeto de buscar estrategias en conjunto y específicas, que disminuyan los tiempos y accesos al examen del VIH, y a las consejerías. Cabe destacar que durante el año 2012, desde el MINSAL, se efectuó un cambio de normativa con respecto a las consejerías, dejando solo las consejerías posteriores al examen como obligatorias, y ampliando el número de profesionales que podían realizar la consejería de pre test, lo que facilitaría el acceso de los usuarios al examen.

\section{REFERENCIAS}

1. Ley 19.779 y Reglamento del Examen para la Detección del Virus de Inmunodeficiencia humana, Decreto No 182 de 2005.

2. MINSAL. Guía clínica: Síndrome de in- 
munodeficiencia adquirida VIH/SIDA. Rev Chilena Infectol. 2010; 27(3): 239-76.

3. Wolff MJ, Cortés CP, Shepherd BE, Beltrán CJ. Long-term outcomes of a national expanded access program to antiretroviral therapy: the Chilean AIDS cohort. J Acquir Immune Defic Syndr. 2010 Nov 1; 55(3): 368-74.

4. Ndiaye B, Salleron J, Vincent A, Bataille P, Bonnevie F, Choisy P, et al. Factors associated with presentation to care with advanced HIV disease in Brussels and Northern France: 1997-2007. BMC Infect Dis. 2011; 11: 11.

5. Couturier E, Schwoebel V, Michon C, Hubert JB, Delmas MC, Morlat P, et al. Determinants of delayed diagnosis of HIV infection in France, 1993-1995. AIDS. 1998 May 7; 12(7): 795-800.

6. MINSAL. Informe nacional sobre los progresos realizados en la aplicación del UNGASS Chile. Informe Final. Santiago: Gobierno de Chile; 2010. p. 135.

7. Schwarcz S, Richards TA, Frank H, Wenzel C, Chin Hsu L, Chin CS, et al. Identifying barriers to HIV testing: personal and contextual factors associated with late HIV testing. AIDS Care. 2011 Mar 18: 1-9.

8. Chadborn TR, Delpech VC, Sabin CA, Sinka K, Evans BG. The late diagnosis and consequent short-term mortality of HIV-infected heterosexuals (England and Wales, 2000-2004). AIDS. 2006; 20(18): 2371-9.

9. Sterling TR, Chaisson RE, Keruly J, Moore RD. Improved outcomes with earlier initiation of highly active antiretroviral therapy among human immunodeficiency virus infected patients who achieve durable virologic suppression: longer follow-up of an observational cohort study. J Infect Dis. 2003; 188(11): 1659-65.

10. Wolff MJ, Beltrán CJ, Vásquez P, Ayala MX, Valenzuela $M$, Berríos $G$, et al. The Chilean AIDS cohort: A model for evaluating the impact of an expanded access program to antiretroviral therapy in a middle-income country-organization and preliminary results. J Acquir Immune Defic Syndr. 2005; 40(5): 551-7.

11. Marks G, Crepaz N, Janssen RS. Estimating sexual transmission of HIV from persons aware and unaware that they are infected with the virus in the USA. AIDS. 2006; 20(10): 1447-50.

12. Lautert L, Dal Pai D, Souza F. Metodología de los estudios exploratorios en investigación de enfermería. En: Do Prado ML, De Souza ML, Montecelli M, Cometto MC, Gómez P. Investigación cualitativa en enfermería. Metodología y didáctica. Washington, DC. OPS; 2013. p. 88-99.

13. Krippendorff K. Content analysis: An introduction to its methodology Thousands Oaks: Sage Publications; 2004.

14. Guba E, Lincoln Y. Competing paradigma in qualitative research.In Denzin N, Lincoln Y. Hand book of qualitative research. Thousand Oask: Sage Publication; 1994, 105-117.

15. Emmanuel E, Wendler D, Grady C. What makes clinical research ethical? JAMA 2000; 283(20): 2701-2711.

16. Jurgensen M, Tuba M, Fylkesnes K, Blystad A. The burden of knowing: balancing benefits and barriers in HIV testing decisions. A qualitative study from Zambia. BMC Health Serv Res. 2012; 12: 2.

17. Daftary A, Padayatchi N, Padilla M. HIV testing and disclosure: A qualitative analysis of TB patients in South Africa. AIDS Care - Psychological and SocioMedical Aspects of AIDS/HIV. 2007; 19(4): 572-7.

18. Ransom JE, Siler B, Peters RM, Maurer MJ. Worry: Women's experience of HIV testing. Qualitative Health Research. 2005; 15(3): 382-93.

19. Sambisa W, Curtis S, Mishra V. AIDS stigma as an obstacle to uptake of HIV 
testing: Evidence from a Zimbabwean national population-based survey. AIDS Care - Psychological and Socio-Medical Aspects of AIDS/HIV. 2010; 22(2): 17086.

20. Spielberg F, Kurth A, Gorbach PM, Goldbaum G. Moving from apprehension to action: HIV counseling and testing preferences in three at-risk populations. AIDS Educ Prev. 2001 Dec; 13(6): 524-40.

21. Nyanzi-Wakholi B, Lara AM, Watera C, Munderi P, Gilks C, Grosskurth H. The role of HIV testing, counselling, and treatment in coping with HIV/AIDS in Uganda: a qualitative analysis. AIDS Care. 2009 Jul; 21(7): 903-8.

22. Mill JE, Jackson RC, Worthington CA, Archibald CP, Wong T, Myers T, et al. HIV testing and care in Canadian aboriginal youth: A community based mixed methods study. BMC Infectious Diseases. $2008 ; 8$.

23. Trieu SL. Partner communication and factors associated with the decision to obtain an HIV Test among Chinese/Chinese American community college students in Northern California [Doctoral thesis]. [California]: Loma Linda University; 2008. $210 \mathrm{p}$.

24. Castle S. Doubting the existence of AIDS: A barrier to voluntary HIV resting and counselling in urban Mali. Health Policy Planning 2003; 18(2): 146-55.

25. MINSAL. Guías Clínicas: Síndrome de
Inmunodeficiencia Adquirida VIH/SIDA. 2010; 97-103.

26. Mitra D, Jacobsen M, O'Connor A, Pottie $\mathrm{K}$, Tugwell P. Assessment of the decision support needs of women from HIV endemic countries regarding voluntary HIV testing in Canada. Patient Educ Couns. 2006; 63(3): 292-300.

27. Barnabas Njozing N, Edin KE, Hurtig AK. 'When I get better I will do the test': Facilitators and barriers to HIV testing in Northwest Region of Cameroon with implications for TB and HIV/AIDS control programmes. SAHARA J : journal of Social Aspects of HIV/AIDS Research Alliance / SAHARA, Human Sciences Research Council. 2010; 7(4): 24-32.

28. Yazdanpanah Y, Lange J, Gerstoft J, Cairns G. Earlier testing for HIV-how do we prevent late presentation? Antivir Ther. 2010; 15 Suppl 1: 17-24.

29. Montaner J. Hay que normalizar la actitud de la gente ante la prueba del sida [Internet]. España: Agencia SINC; sección Biomedicina y salud: Ciencia clínicas; 2013 [citado 28 ene 2014]. Disponible en: http://www.agenciasinc.es/Entrevistas/ Hay-que-normalizar-la-actitud-de-lagente-ante-la-prueba-del-sida

30. Irarrazábal L, Ferrer L, Cianelli C, Lara L, Reed R, Levy J, et al. Prueba oral rápida: una alternativa al tamizaje tradicional del VIH en Chile. Rev Panam Salud Publica. 2013; 33(6): 427-3. 